\title{
Microbiological Profile of Infectious Keratitis in a Portuguese Tertiary Centre
}

\author{
Cláudia Oliveira-Ferreira $\left(\mathbb{D}^{\mathrm{D}},{ }^{1}\right.$ Mariana Leuzinger-Dias, ${ }^{1}$ João Tavares-Ferreira ${ }^{(\mathbb{D})}{ }^{1}$ \\ Luís Torrão, ${ }^{1}$ and F. Falcão-Reis ${ }^{1,2}$ \\ ${ }^{1}$ Ophthalmology Department, Centro Hospitalar São João, Porto, Portugal \\ ${ }^{2}$ Department of Surgery and Physiology, Faculty of Medicine of Porto University, Porto, Portugal
}

Correspondence should be addressed to Cláudia Oliveira-Ferreira; mofclaudia@gmail.com

Received 22 July 2019; Accepted 25 September 2019; Published 24 October 2019

Academic Editor: Enrique Mencía-Gutiérrez

Copyright (C) 2019 Cláudia Oliveira-Ferreira et al. This is an open access article distributed under the Creative Commons Attribution License, which permits unrestricted use, distribution, and reproduction in any medium, provided the original work is properly cited.

\begin{abstract}
The microbiological profile of infectious keratitis has shown great differences across the world. Due to the continuous shifting trends in microbiological profile and antibiotic resistance patterns reported in several studies, constant local updates are crucial to provide an adequate treatment. The propose of this study was to analyze the incidence of infectious keratitis, possible changing trends in microbiological profile, and bacteria sensitivity to commonly used antibiotics, in our tertiary center, in the last 10 years. A retrospective study was performed, based on the survey review of electronic medical records of all patients with presumed infectious keratitis, between January 1, 2009, and December 31, 2018. Microbial cultures were performed, and patients were treated according to an internal protocol. A total of 1360 samples were included. We obtained a $35.1 \%$ culture-positive rate. Bacteria accounted for $76.78 \%$ of all positive scrapes (53.34\% were Gram positive and $23.44 \%$ were Gram negative), Acanthamoeba for $12.13 \%$, fungi for $8.16 \%$, and virus for $2.93 \%$. The most frequent agent identified was Corynebacterium macginleyi (18.41\%), followed by Staphylococcus aureus (17.78\%), Streptococcus pneumoniae (9.41\%), and Pseudomonas aeruginosa (9.00\%). We identified at least one ophthalmologic risk factor in 410 patients $(85.77 \%)$. Trauma and contact lens wear were the most common risk factors found, accounting for $34.94 \%(n=167)$ and $33.47 \%(n=160)$ of cases. Sensitivity to fluoroquinolones and aminoglycosides was tested in all bacterial isolates, presenting values of $96.66 \%$ and $98.12 \%$. In our region, the most common bacteria are Staphylococcus aureus, Streptococcus pneumoniae, and Pseudomonas aeruginosa, and they showed high sensitivity rates to first-line antibiotics, without any modification or emergence of antibiotic resistance trends during the 10 years of the study. For this reason, we decided to maintain the same internal protocol in our tertiary centre.
\end{abstract}

\section{Introduction}

Infectious keratitis is a serious and frequent cause of ophthalmology consultation and one of the greatest causes of visual impairment worldwide $[1,2]$. The incidence of this pathology is highly variable around the world. It is estimated to affect about 11/100,000 inhabitants in the United States, rising to 799/100,000 inhabitants in developing countries like Nepal [3,4].

Infectious keratitis is associated with significant morbidity, carrying a high risk of corneal scar, corneal perforation, endophthalmitis, and vision loss.
Contact lens usage and ocular trauma are the major risk factors to develop infectious keratitis. However, other conditions such as ocular surface diseases (blepharitis and dry eye) and systemic disorders (diabetes, rheumatoid arthritis, and acquired immunodeficiency syndrome) are also strongly associated with the onset of this disease [5].

Clinically, it manifests as eye pain, photophobia, blurred vision, and ciliary injection.

The microbiological profile of infectious keratitis has shown great differences across the world, but bacteria triumph as the leading cause of infectious keratitis, followed by fungi (especially in cases of ocular trauma with 
vegetable matter). Virus and protozoa are less frequent agents $[6,7]$.

Having in mind this etiological pattern, broad-spectrum antibiotics are used as empiric first-line treatment for presumed infectious keratitis, after obtaining corneal scrapes [2]. However, to maintain the effectiveness of empiric therapy, it is necessary to assure low resistance rates to the selected antibiotics. Due to the widespread use of broadspectrum antibiotics, and continuous shifting trends in microbiological profile and antibiotics resistance profiles reported in several studies, constant local updates of microbiological profile and antibiotic resistance patterns are crucial to provide an adequate treatment [8-13].

The propose of this study was to analyze the incidence of infectious keratitis, possible changing trends in microbiological profile, and bacteria sensitivity to commonly used antibiotics, in our tertiary center, in the last 10 years.

\section{Materials and Methods}

This retrospective study was performed in a Portuguese tertiary center, based on the survey review of electronic medical records of all patients with presumed infectious keratitis, between January 1, 2009, and December 31, 2018. Presumed infectious keratitis was defined by the presence of a corneal infiltrate $>1 \mathrm{~mm}^{2}$ in size with or without epithelial defect.

All samples were collected in the emergency room, under topical anesthesia. Every time, scrapes were obtained from two different locations (conjunctival fornix and ulcer base) and then inoculated onto Amies Agar Gel (Copan, Brescia, Italy). Finally, they were sent to the Microbiology Department for culture and antibiotic sensitivity testing. In clinically suspicious or nonresponsive cases, selective media and stains for aerobic and anaerobic bacteria, Mycobacterium and Acanthamoeba, were used.

Patients were treated according to an internal protocol, using an empiric third- or fourth-generation fluoroquinolone and an aminoglycoside, hourly. If a fungi etiology was suspected (soil contamination or trauma with vegetable matter), topical clotrimazole was added to the treatment regimen.

Patients were assessed for age, gender, presence of risk factors (contact lens usage, ocular trauma, ocular surface diseases, or systemic diseases), sample results, and antibiotic sensitivity test results. The study was therefore divided into 2 periods for analysis: from 2009 to 2013 and from 2013 to 2018.

Statistical analysis was performed using SPSS software, version 22 (IBM, Chicago, IL). To compare differences between groups, chi-square test, $t$-test, logistic, and multiple regression were performed, as appropriated. $P<0.05$ was considered significant.

\section{Results}

A total of one thousand three hundred and sixty $(n=1360)$ samples were included. Patients' mean age was $46.88 \pm 20.327$ years (range 2-98), and $50.3 \%$ of them were males. Over the

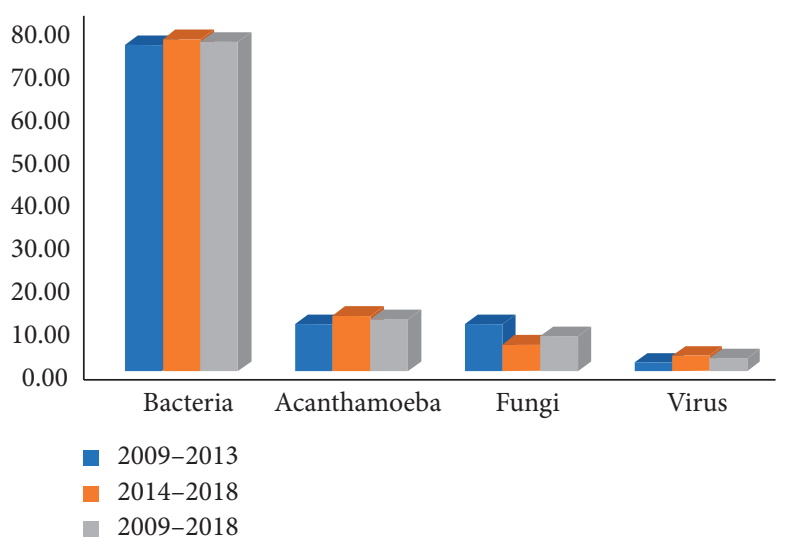

Figure 1: Distribution of microbial profile in the two periods studied.

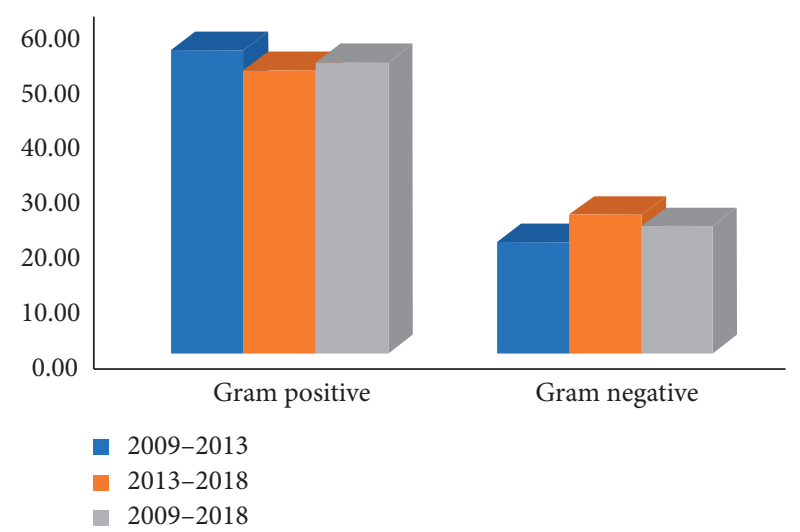

Figure 2: Proportion of Gram-positive and Gram-negative isolates in the two periods studied.

years, we observed an increase in the number of suspected cases of infectious keratitis, with the value doubling in 10 years. We obtained a $35.1 \%(n=478 / 1360)$ culture-positive rate. The percentage of positive microbiological cultures per year ranged from 31.31 to $42.22 \%$. Per period, this value was $37.76 \%$ and $33.71 \%$ in $2009-2013$ and 2014-2018, respectively, without significant statistical differences $(P>0.05)$.

Bacteria accounted for $76.78 \%$ ( $76 \%$ in $2009-2013$; $77.40 \%$ in $2014-2018$ ) of all positive scrapes $(53.34 \%$ were Gram positive and $23.44 \%$ were Gram negative), Acanthamoeba for $12.13 \%$, fungi for $8.16 \%$, and virus for $2.93 \%$ (Figure 1).

Gram-positive bacteria accounted for $55.50 \%$ of all positive scrapes in 2009-2013 and 51.80\% in 2014-2018, while Gram-negative bacteria accounted for $20.50 \%$ and $25.54 \%$ in 2009-2013 and 2014-2018, respectively, without significant statistical changes (Figure 2). The most frequent Grampositive microorganism isolated was Corynebacterium macginleyi (18.41\%), followed by Staphylococcus aureus (17.78\%), Streptococcus pneumonia (9.41\%), and Staphylococcus epidermidis (4.39\%). No significant statistical changes were observed in the microbiological profile in both periods. The most frequent Gram-negative agents were Pseudomonas aeruginosa, Serratia spp., Moraxella spp., and Haemophilus 
TABLE 1: Distribution of microbial profile.

\begin{tabular}{lcc}
\hline & $n=478$ & $\%$ \\
\hline Bacteria & 367 & 76.78 \\
Gram positive & 255 & 53.34 \\
Corynebacterium macginleyi & 88 & 18.41 \\
Staphylococcus aureus & 85 & 17.78 \\
Streptococcus pneumoniae & 45 & 9.41 \\
Staphylococcus epidermis & 21 & 4.39 \\
Streptococcus mitis/oralis & 7 & 1.46 \\
Streptococcus viridans & 7 & 1.46 \\
Streptococcus pyogenes & 1 & 0.21 \\
Kocuria rosea & 1 & 0.21 \\
Gram negative & 112 & 23.44 \\
Pseudomonas aeruginosa & 43 & 9.00 \\
Serratia specimens & 27 & 5.65 \\
Moraxella spp. & 20 & 4.18 \\
Haemophilus influenzae & 7 & 1.46 \\
Enterobacter cloacae & 4 & 0.84 \\
Morganella morganii & 3 & 0.63 \\
Proteus mirabilis & 3 & 0.63 \\
Aeromonas hydrophila & 2 & 0.42 \\
Klebsiella spp. & 1 & 0.21 \\
Kingella kingae & 1 & 0.21 \\
Stenotrophomonas maltophilia & 1 & 0.21 \\
\hline Acanthamoeba & $\mathbf{5 8}$ & $\mathbf{1 2 . 1 3}$ \\
\hline Fungi & $\mathbf{3 9}$ & $\mathbf{8 . 1 6}$ \\
Candida spp. & 20 & 4.18 \\
Fusarium spp. & 7 & 1.46 \\
Aspergillus spp. & 6 & 1.26 \\
Paecilomyces spp. & 3 & 0.63 \\
Scedosporium spp. & 2 & 0.42 \\
Mucor & $\mathbf{1 4}$ & 0.21 \\
\hline Virus & $\mathbf{2 . 9 3}$ \\
\hline
\end{tabular}

TABLE 2: Risk factors.

\begin{tabular}{lcc}
\hline & $n$ & $\%$ \\
\hline Local & $\mathbf{4 1 0}$ & $\mathbf{8 5 . 7 7}$ \\
Ocular trauma & 167 & 34.94 \\
Contact lens use & 160 & 33.47 \\
Blepharitis & 20 & 4.18 \\
Ocular infection & 19 & 3.97 \\
Exposure keratopathy & 13 & 2.72 \\
Dry eye & 10 & 2.09 \\
Bullous/band keratopathy & 8 & 1.67 \\
Recurrent erosion & 7 & 1.46 \\
Trichiasis & 3 & 0.63 \\
Herpetic keratitis history & 3 & 0.63 \\
\hline Systemic & $\mathbf{1 0 5}$ & $\mathbf{2 1 . 9 7}$ \\
Diabetes mellitus & 60 & 12.55 \\
Poor systemic status/multiple comorbidities & 14 & 2.93 \\
Mucocutaneal disease & 13 & 2.72 \\
Autoimmune disease under immunosuppressant & 6 & 1.26 \\
Neoplasia & 6 & 1.26 \\
HIV & 3 & 0.63 \\
Drugs & 3 & 0.63 \\
\hline
\end{tabular}

influenzae $(9.00 \%, 5.65 \%, 4.18 \%$, and $1.46 \%$, respectively). Again, no significant statistical changes were observed in the microbiological profile in both periods.
Acanthamoeba accounted for $11 \%$ and $12.95 \%$ of positive scrapes in 2009-2013 and 2014-2018, respectively, without significant statistical differences in both periods. The same was observed with fungi (11\% and 6.11\% in 2009-2013 and 2014-2018, respectively) and virus (2\% and $3.60 \%$ in 2009-2013 and 2014-2018, respectively). Candida specimens, Fusarium specimens, and Aspergillus specimens accounted for $4.18 \%, 1.46 \%$, and $1.26 \%$ of all positive scrapes. The distribution of microbial profile is shown in Table 1.

We identified at least one ophthalmologic risk factor in 410 patients $(85.77 \%)$. Additional systemic disorders were present in $17.57 \%(n=84)$. Trauma and contact lens wear were the most common risk factors found, accounting for $34.94 \%(n=167)$ and $33.47 \%(n=160)$ of cases. The distribution of the risk factors is demonstrated in Table 2.

Sensitivity testing was not performed for $\mathrm{Co}$ rynebacterium macginleyi, as the laboratory considered it as a contaminant, but it was performed in all the remaining bacterial isolates.

Sensitivity to fluoroquinolones and aminoglycosides was tested in all bacterial isolates, presenting values of $96.66 \%$ and $98.12 \%$, respectively, with no significant statistical differences between the periods analyzed. The sensitivity to antibiotics is demonstrated in Table 3.

Staphylococcus aureus showed a high sensitivity profile to fluoroquinolones (ciprofloxacin, moxifloxacin, and levofloxacin) and to aminoglycosides (gentamicin and tobramycin), 89.41\% and 98.82\%, respectively. Two cases were methicillin-resistant Staphylococcus aureus (MRSA), being sensitive to vancomycin and linezolid only. All Streptococcus pneumonia isolates were sensitive to fluoroquinolones and aminoglycosides, and only a small proportion (4.44\%) was resistant to tetracyclines. $85.72 \%$ of Staphylococcus epidermidis isolates were sensitive to fluoroquinolones and the same proportion to aminoglycosides, but resistance to tetracycline was observed in $38.09 \%$ of cases.

Pseudomonas aeruginosa presented a sensitivity of 95.35\% to fluoroquinolones and aminoglycosides, and 27.91\% demonstrated resistance to cotrimoxazole. Besides, $4.35 \%$ of cases showed multidrug resistance (resistance to at least three different classes of antibiotics), being sensitive exclusively to cefepime and colistin. All Serratia specimens and Moraxella catarrhalis isolates were sensitive to fluoroquinolones and aminoglycosides. No differences were observed in sensitivity to antibiotics in both periods analyzed.

\section{Discussion/Conclusion}

Considering the widespread use of broad-spectrum antibiotics, antibiotic resistance among bacterial microorganisms in general is a real concern nowadays, and ocular pathogens are no exception. Therefore, constant local updates of microbiological profiles and antibiotic resistance patterns are crucial to wisely choose the most appropriate topical treatment to ocular infections.

Infectious keratitis is a serious condition that can lead to a poor visual outcome or even loss of the eyeball. The 
TABLe 3: Sensitivity to fluoroquinolones and aminoglycosides.

\begin{tabular}{|c|c|c|c|c|}
\hline & Bacteria & $n$ & Sensitivity to fluoroquinolones (\%) & Sensitivity to aminoglycosides (\%) \\
\hline \multirow{5}{*}{ Gram positive } & Staphylococcus aureus & 85 & 89.41 & 98.82 \\
\hline & Streptococcus pneumoniae & 45 & 100 & 100 \\
\hline & Staphylococcus epidermis & 21 & 85.72 & 85.72 \\
\hline & Streptococcus mitis/oralis & 7 & 100 & 71.43 \\
\hline & Streptococcus viridans & 7 & 100 & 71.43 \\
\hline \multirow{4}{*}{ Gram negative } & Pseudomonas aeruginosa & 43 & 95.35 & 95.35 \\
\hline & Serratia specimens & 27 & 100 & 100 \\
\hline & Moraxella spp. & 20 & 100 & 100 \\
\hline & Haemophilus influenzae & 7 & 100 & 100 \\
\hline
\end{tabular}

diversity of the clinical presentation constitutes a huge challenge to an accurate diagnosis and treatment. Untreated keratitis can lead to opacification and/or perforation of the cornea, as well as to other equally fearsome complications such as secondary glaucoma, corneal thinning, uveitis, and endophthalmitis. Due to this rapidly progressive and potentially devastating course, an appropriate treatment with effective empirical topical antibiotics is mandatory.

It is well known that the epidemiological pattern of infectious keratitis significantly varies between countries and even among regions of the same country [8-13]. Therefore, determining the local microbiological profile, of one specific region or country, as well as its antibiotic resistance patterns and trends over the years is of major interest to achieve an effective therapeutic strategy [13-15].

In this study, we analyzed a total of 1360 corneal scrapes obtained from patients diagnosed with infectious keratitis, admitted to a tertiary hospital in Portugal.

We obtained a $35.1 \%(n=478 / 1360)$ culture-positive rate, a result in agreement with similar studies published in the literature $[14,16,17]$.

In our study, bacteria were the agent isolated in the vast majority of cases. Gram-positive bacteria were identified in $53.34 \%$ of the positive cultures, a value that is similar to other studies already published $[14,17]$. However, in countries like the United Kingdom or New Zealand, proportions of $38.9 \%$ and $83 \%$, respectively, have been reported [16, 18]. A Canadian study reported a decreasing trend in Gram-positive pathogens, probably due to the generalization of contact lens usage [13]. However, in our study, no statistically significant differences between Gram-positive and Gram-negative bacteria were observed between the 2 analyzed periods.

The most frequent agent identified was Corynebacterium macginleyi (18.41\%), followed by Staphylococcus aureus (17.78\%), Streptococcus pneumoniae, (9.41\%) and Pseudomonas aeruginosa (9.00\%). In the literature, some studies showed Pseudomonas aeruginosa as the most frequent agent $[13,14,16,18]$, while in other studies, Staphylococcus aureus is the main causal agent $[13,14,16]$. In our hospital, Corynebacterium macginleyi was considered by the Microbiological Department as a scrape contaminant. Nevertheless, it seems to actively contribute to the pathophysiology of blepharitis, and thus, it can potentially play a role in the genesis of infectious keratitis, particularly if some risk factors are present [19]. For this reason, its importance as a causative agent of infections has been progressively taken into account [20-22].

Acanthamoeba accounted for $12.13 \%$ of positive scrapes, and this value is located between the ranges published in the literature $(1.6 \%$ and $16.9 \%)[1,23]$.

Fungi represented $8.16 \%$ of positive scrapes, Candida spp. being the most frequent. However, the incidence of fungi is much higher in some countries such as Brazil (30\%) or India (23-36\%) since these microbiological agents are more frequent in tropical and subtropical regions than in the temperate regions $[1,24,25]$.

Globally, fluoroquinolone sensitivity was high (96.66\%), and the same was observed with aminoglycoside sensitivity (98.12\%). Although these results are similar to several other series already published, [13-16, 18] resistances to fluoroquinolone and aminoglycosides of about $15 \%$ and $22 \%$ have been reported in Switzerland [26]. In our region, fluoroquinolones and aminoglycosides are almost exclusively prescribed by ophthalmologists. In primary health care, chloramphenicol (eye drops and ointment) and oxytetracycline (ointment) are the most widely used drugs. This probably explains why patients naive to infectious keratitis have low resistance rates.

Infectious keratitis caused by MRSA is an alarming issue all around the world due to its poor response to conventional antibiotic treatment [27-29]. Our rate of MRSA was $0.42 \%$, lower than other reported series [2]. Like in other studies previously published, we observed that the rate of MRSA sensitivity to vancomycin was $100 \%$, and therefore, vancomycin is a drug of inestimable value for the treatment of MRSA infections [30-34].

In our Microbiological Department, Corynebacterium macginley was considered a scrape contaminant. In the first reports in the literature, this agent was highly sensitive to common topical antibiotics, but this scenario has now changed, and high levels of resistance to fluoroquinolones have been already reported [35-37].

Some of the differences found in our study, compared to others already published, can be explained by factors such as the geographic location and associated climate and the extent and type of empirical antimicrobial therapy instituted before corneal scrapes. In our region, the most common bacteria are Staphylococcus aureus, Streptococcus pneumoniae, and Pseudomonas aeruginosa, and they showed high sensitivity rates to first-line antibiotics, without any modification or emergence of antibiotic resistance trends during 
the 10 years of the study. For this reason, we decided to maintain the same internal protocol in our tertiary centre.

\section{Data Availability}

The data used to support the findings of this study are available from the corresponding author upon request.

\section{Conflicts of Interest}

The authors declare that there are no conflicts of interest regarding the publication of this paper.

\section{References}

[1] R. Farias, L. Pinho, and R. Santos, "Epidemiological profile of infectious keratitis," Revista Brasileira de Oftalmologia, vol. 76, no. 3, pp. 116-120, 2017.

[2] S. Mediero, A. Boto de los Bueis, K. Spiess et al., "Clinical and microbiological profile of infectious keratitis in an area of Madrid, Spain," Enfermedades Infecciosas Y Microbiología Clínica, vol. 36, no. 7, pp. 409-416, 2018.

[3] J. C. Eric, M. P. Nevitt, D. O. Hodge, and D. J. Ballard, "Incidence of ulcerative keratitis in a defined population from 1950-1988," Archives of Ophthalmology, vol. 111, no. 12, pp. 1665-1671, 1993.

[4] M. P. Upadhyay, P. C. Karmacharya, S. Koirala et al., "The Bhaktapur eye study: ocular trauma and antibiotic prophylaxis for the prevention of corneal ulceration in Nepal," British Journal of Ophthalmology, vol. 85, no. 4, pp. 388-392, 2001.

[5] R. S. Sacramento, L. B. Souza, E. H. Sato et al., "Estudos dos fatores epidemiológicos e influentes na ceratite microbiana em serviço universitário," Revista Brasileira de Oftalmologia, vol. 64, no. 1, pp. 7-13, 2005.

[6] M. R. Alves and B. B. A. d. Andrade, "Úlcera de córnea bacteriana," Arquivos Brasileiros de Oftalmologia, vol. 63, no. 6, pp. 495-498, 2000.

[7] E. Wakisaka, M. A. Ferreira, F. J. d. Rocha, L. d. L. Freitas, T. Guidugli, and A. L. H. d. Lima, "Cultura de material provindo de úlcera de córnea em laboratório de referência," Arquivos Brasileiros de Oftalmologia, vol. 53, no. 5, pp. 193198, 1990.

[8] A. Shah, A. Sachdev, D. Coggon, and P. Hossain, "Geographic variations in microbial keratitis: an analysis of the peerreviewed literature," British Journal of Ophthalmology, vol. 95, no. 6, pp. 762-767, 2011.

[9] S. Sharma, D. Y. Kunimoto, U. Gopinathan, S. Athmanathan, P. Garg, and G. N. Rao, "Evaluation of corneal scraping smear examination methods in the diagnosis of bacterial and fungal keratitis," Cornea, vol. 21, no. 7, pp. 643-647, 2002.

[10] G. Alexandrakis, E. C. Alfonso, and D. Miller, "Shifting trends in bacterial keratitis in South Florida and emerging resistance to fluoroquinolones," Ophthalmology, vol. 107, no. 8, pp. 1497-1502, 2000.

[11] H. O. Orlans, S. J. Hornby, and I. C. J. W. Bowler, "In vitro antibiotic susceptibility patterns of bacterial keratitis isolates in Oxford, UK: a 10-year review," Eye, vol. 25, no. 4, pp. 489-493, 2011.

[12] X. Sun, S. Deng, R. Li et al., "Distribution and shifting trends of bacterial keratitis in north China (1989-98)," British Journal of Ophthalmology, vol. 88, no. 2, pp. 165-166, 2004.
[13] A. Lichtinger, S. N. Yeung, P. Kim et al., "Shifting trends in bacterial keratitis in toronto," Ophthalmology, vol. 119, no. 9, pp. 1785-1790, 2012.

[14] A. L.-K. Ng, K. K.-W. To, C. C.-L. Choi et al., "Predisposing factors, microbial characteristics, and clinical outcome of microbial keratitis in a tertiary centre in Hong Kong: a 10-year experience," Journal of Ophthalmology, vol. 2015, Article ID 769436, 9 pages, 2015.

[15] D. S. C. Lam, E. Houang, D. S. P. Fan, D. Lyon, D. Seal, and E. Wong, "Incidence and risk factors for microbial keratitis in Hong Kong: comparison with Europe and North America," Eye, vol. 16, no. 5, pp. 608-618, 2002.

[16] Z. Shalchi, A. Gurbaxani, M. Baker, and J. Nash, "Antibiotic resistance in microbial keratitis: ten-year experience of corneal scrapes in the United Kingdom," Ophthalmology, vol. 118, no. 11, pp. 2161-2165, 2011.

[17] J. Hong, J. Xu, J. Hua, and X. Sun, "Bacterial keratitis in Shanghai," Ophthalmology, vol. 120, no. 3, p. 647, 2013.

[18] A. Pandita and C. Murphy, "Microbial keratitis in Waikato, New Zealand," Clinical \& Experimental Ophthalmology, vol. 39, no. 5, pp. 393-397, 2011.

[19] C. S. Ferreira, L. Figueira, N. Moreira-Gonçalves et al., Corynebacterium Macginleyi in Corneal Ulcers-Pathogen or Contaminant?, 5th Eucornea, London, UK, 2014.

[20] C. Zhang, Y. Liang, S. Deng, Z. Wang, R. Li, and X. Sun, "Distribution of bacterial keratitis and emerging resistance to antibiotics in China from 2001 to 2004," Clinical Ophthalmology, vol. 2, pp. 575-579, 2008.

[21] I. Bezza Benkaouha, C. Le Brun, P.-J. Pisella, J. Chandenier, and P. Lanotte, "La flore bactérienne dans les blépharites," Journal Français d'Ophtalmologie, vol. 38, no. 8, pp. 723-728, 2015.

[22] K. L. Ruoff, C. M. Toutain-Kidd, M. Srinivasan et al., "Corynebacterium macginleyi isolated from a corneal ulcer," Infectious Disease Reports, vol. 2, no. 1, 2010.

[23] A. M. Otri, U. Fares, M. A. Al-Aqaba et al., "Profile of sightthreatening infectious keratitis: a prospective study," Acta Ophthalmologica, vol. 91, no. 7, pp. 643-651, 2013.

[24] P. A. Sedhu, S. Sugathan, A. Pushpakaran, and C. Kurian, "Bacterial and fungal profile of infectious keratitis: a prospective study," International Journal of Scientific Study, vol. 5, no. 8, pp. 128-132, 2017.

[25] R. Priyamvada, D. Shukla, N. P. Singh et al., "Changing trends in fungal and bacterial profile of infectious keratitis at a tertiary care hospital: a six-year study," Clinical Epidemiology and Global Health, vol. 5, no. 1, pp. 40-45, 2017.

[26] F. Schaefer, O. Bruttin, L. Zografos, and L. Guex-Crosier, "Bacterial keratitis: a prospective clinical and microbiological study," British Journal of Ophthalmology, vol. 85, no. 7, pp. 842-847, 2001.

[27] K. J. Wroblewski, J. F. Pasternak, K. S. Bower et al., "Infectious keratitis after photorefractive keratectomy in the United States army and navy," Ophthalmology, vol. 113, no. 4, pp. 520-525, 2006.

[28] R. Solomon, E. D. Donnenfeld, H. D. Perry et al., "Methicillinresistant Staphylococcus aureus infectious keratitis following refractive surgery," American Journal of Ophthalmology, vol. 143, no. 4, pp. 629-634, 2007.

[29] C. Sotozono, K. Inagaki, A. Fujita et al., "Methicillin-resistant Staphylococcus aureus and methicillin-resistant Staphylococcus epidermidis infections in the cornea," Cornea, vol. 21, pp. 94-101, 2002.

[30] P. A. C. Maple, J. M. T. Hamilton-Miller, and W. Brumfitt, "World-wide antibiotic resistance in methicillin-resistant 
Staphylococcus aureus," The Lancet, vol. 333, no. 8637, pp. 537-540, 1989.

[31] N. Cimolai, "Ciprofloxacin and multiresistant staphylococci," The Lancet, vol. 349, no. 9057, p. 1030, 1997.

[32] M. Maffett and D. M. O’Day, "Ciprofloxacin-resistant bacterial keratitis," American Journal of Ophthalmology, vol. 115, no. 4, pp. 545-546, 1993.

[33] F. B. Marangon, D. Miller, M. S. Muallem, A. C. Romano, and E. C. Alfonso, "Ciprofloxacin and levofloxacin resistance among methicillin-sensitive Staphylococcus aureus isolates from keratitis and conjunctivitis," American Journal of Ophthalmology, vol. 137, no. 3, pp. 453-458, 2004.

[34] A. F. Elsahn, E. H. Yildiz, D. L. Jungkind et al., "In vitro susceptibility patterns of methicillin-resistant Staphylococcus aureus and coagulase-negative staphylococcus corneal isolates to antibiotics," Cornea, vol. 29, no. 10, pp. 1131-1135, 2010.

[35] A. M. Joussen, G. Funke, F. Joussen, and G. Herbertz, "Corynebacterium macginleyi: a conjunctiva specific pathogen," British Journal of Ophthalmology, vol. 84, no. 12, pp. 14201422, 2000.

[36] H. Eguchi, T. Kuwahara, T. Miyamoto et al., "High-level fluoroquinolone resistance in ophthalmic clinical isolates belonging to the species Corynebacterium macginleyi," Journal of Clinical Microbiology, vol. 46, no. 2, pp. 527-532, 2008.

[37] A. R. Alsuwaidi, D. Wiebe, T. Burdz et al., "Corynebacterium macginleyi conjunctivitis in Canada," Journal of Clinical Microbiology, vol. 48, no. 10, pp. 3788-3790, 2010. 


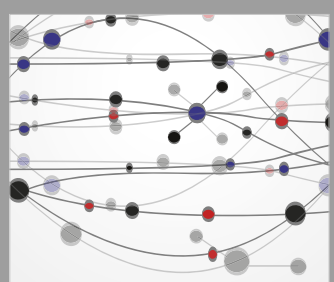

The Scientific World Journal
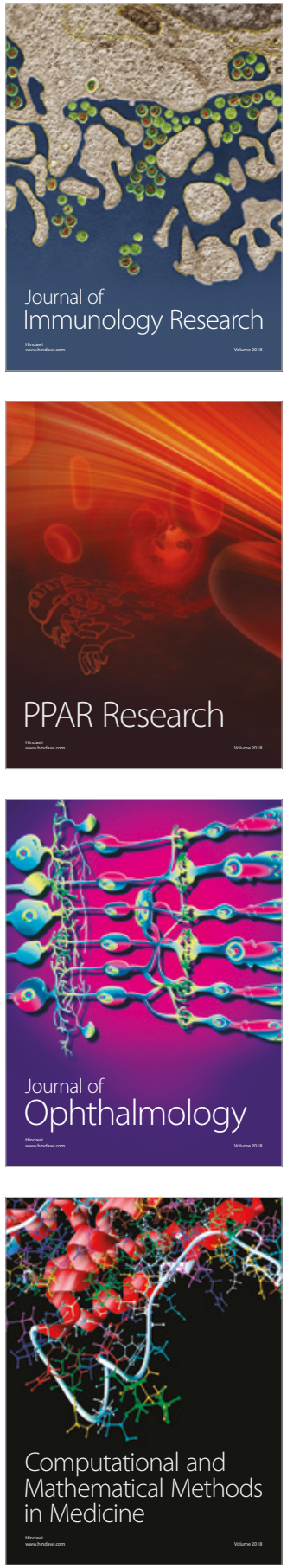

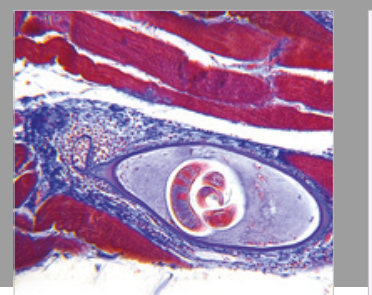

Gastroenterology Research and Practice

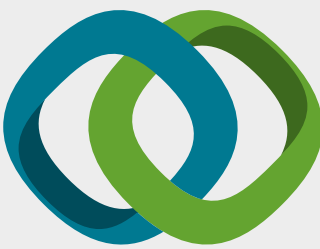

\section{Hindawi}

Submit your manuscripts at

www.hindawi.com
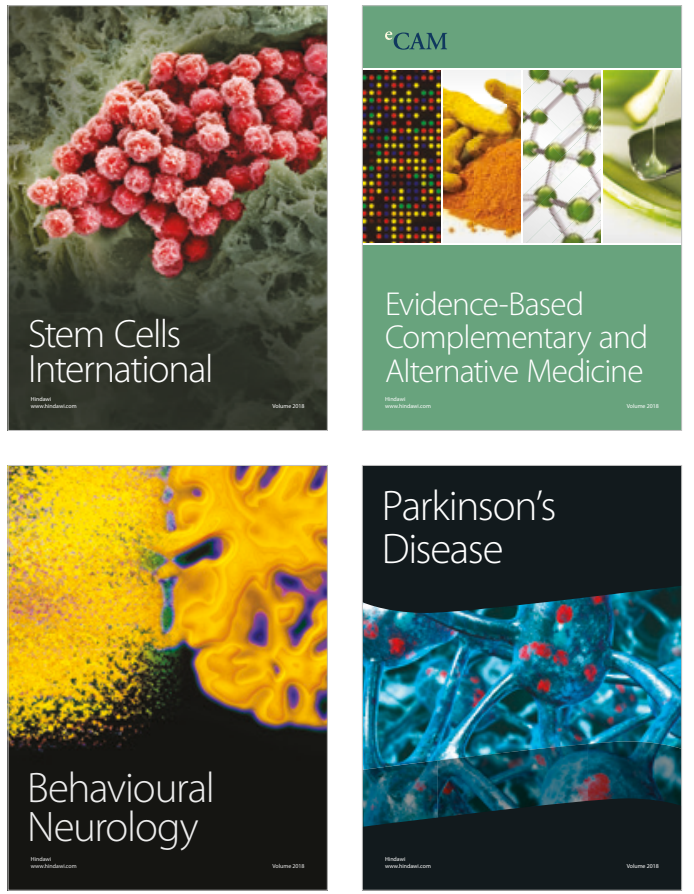

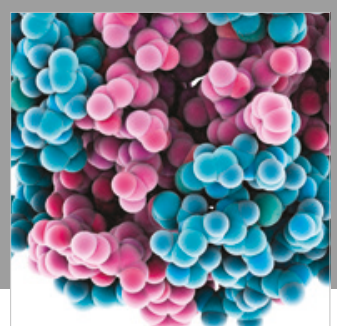

ournal of

Diabetes Research

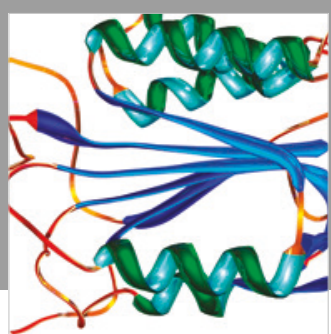

Disease Markers
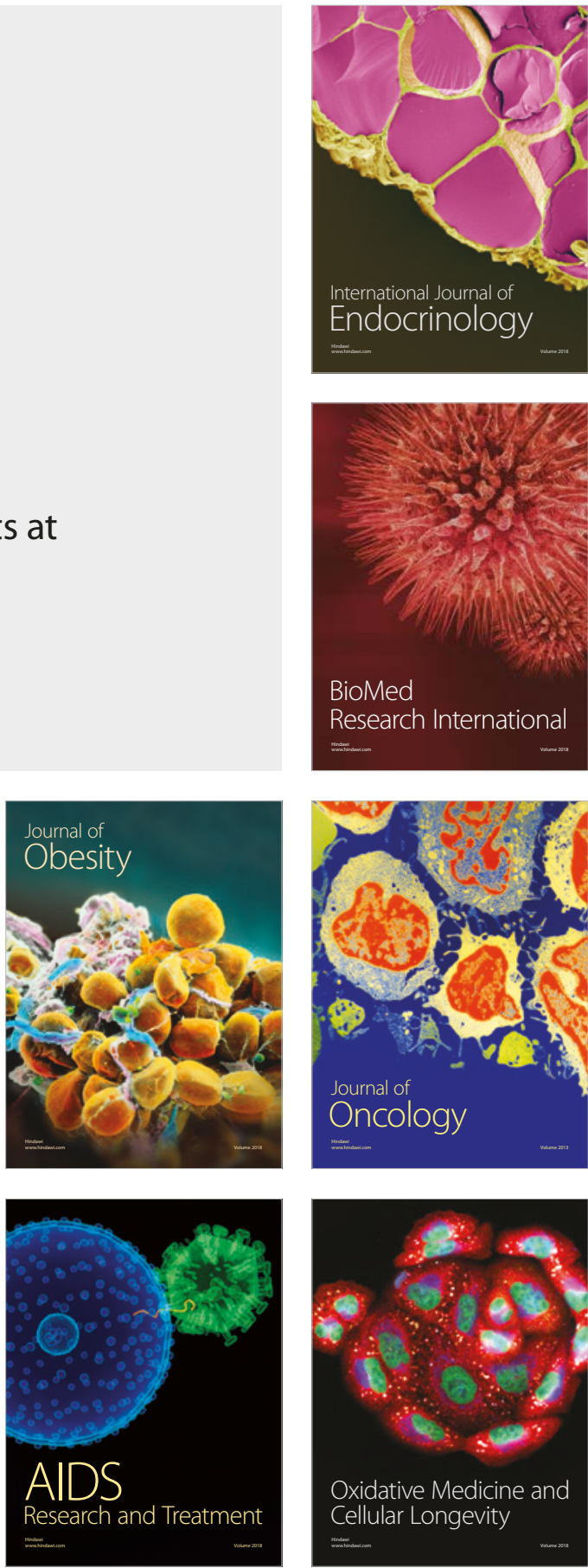